\title{
Equivalent circuit models for electronic ceramics
}

\author{
LAKSHMAN PANDEY ${ }^{\dagger \dagger}$, OM PARKASH, RAJESH K KATARE ${ }^{\dagger \dagger}$ and \\ DEVENDRA KUMAR ${ }^{\dagger}$ \\ School of Materials Science and Technology, ${ }^{+}$Department of Ceramic Engineering, Institute \\ of Technology, Banaras Hindu University, Varanasi 221005 , India \\ ${ }^{\star}$ Department of Physics, Rani Durgavati University, Jabalpur 482001, India \\ MS received 25 August 1994; revised 23 May 1995
}

\begin{abstract}
Complex immittance spectra of model equivalent circuits involving resistive and capacitive elements are calculated. A comparison of experimentally obtained complex immittance plots with these diagrams greatly facilitates the search for the most appropriate equivalent circuit representing the electrical properties of electronic ceramics.
\end{abstract}

Keywords. Electronic ceramics; immittance spectroscopy; impedance; admittance; modulus.

\section{Introduction}

The electrical properties of electronic ceramics are a result of different contributions from various components and processes present in the material. In general the overall dielectric properties arise due to intra-grain, inter-grain and electrode processes. The motion of charges could take place in any fashion viz. charge displacement, dipole reorientation, space charge formation etc. (Von Hipple 1954; Macdonald 1987). In order to achieve reproducibility and to have a control over the properties, these so-called grain, grain-boundary and electrode contributions must be separated out.

The method of complex impedance analysis (CIA) (Macdonald 1987) has emerged as a very powerful tool for separating out these contributions. In this method the imaginary part, $Z^{\prime \prime}$, of the total complex impedance,

$$
Z^{*}=Z^{\prime}-j Z^{\prime \prime} \text {, }
$$

of the sample is plotted as a function of the corresponding real part, $Z^{\prime}$, at different frequencies. This plot shows different features depending upon various relative contributions. For example, for an electronic ceramic having well separated relaxation times for grain, grain-boundary and electrode contributions, three distinct semi-circular arcs are obtained and for two contributions, two arcs are obtained (Hodge et al 1976; Hooper 1977; Gerhardt and Nowick 1986; Macdonald 1987; Parkash et al 1990). The analysis is carried out further by representing and visualizing these contributing processes by simple equivalent circuits involving lumped elements to start with. Then the values of these components are determined by finding the intercepts on the $Z^{\prime}$ axes (Macdonald 1987) or by using the method of complex non-linear least squares (Macdonald and Garber 1977; Macdonald et al 1982; Macdonald 1987; Pandey 1992, 1993).

A general problem encountered here is the choice of a simple circuit that could represent the experimental data best. In this paper, we present the calculated impedance $\left(Z^{\prime \prime}\right.$ vs $Z^{\prime}$ ) admittance ( $Y^{\prime \prime}$ vs $Y^{\prime}$ where $Y^{*}=1 / Z^{*}$ ) and modulus plots ( $M^{\prime \prime}$ vs $M^{\prime}$ where $M=j \omega C o Z^{*}$ ) of equivalent circuits that are suitable for representing two processes only, having a wide range of ratio of relaxation times. 

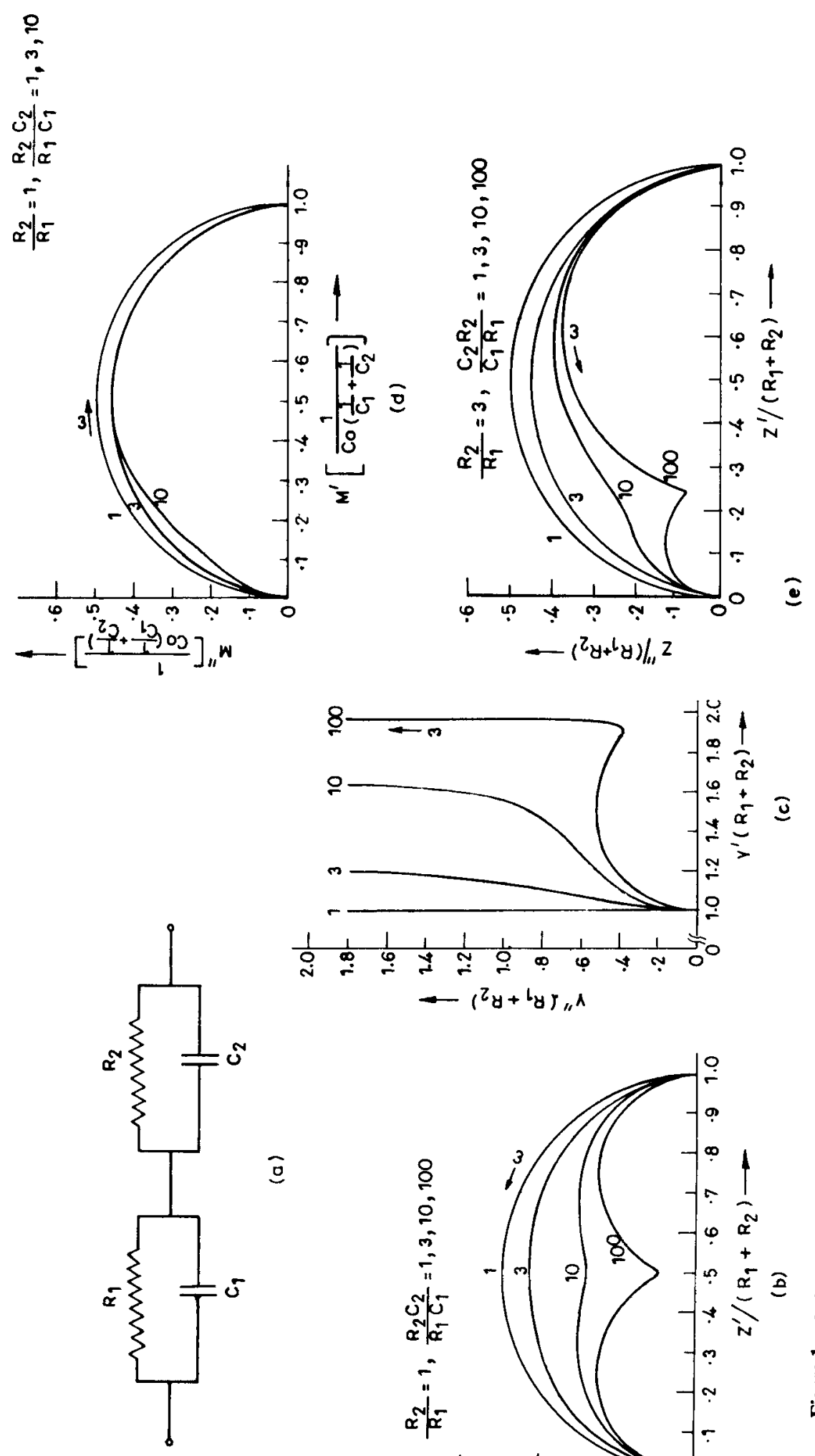

$$
-\left(z_{y}+b_{y}\right)_{1,} 1
$$

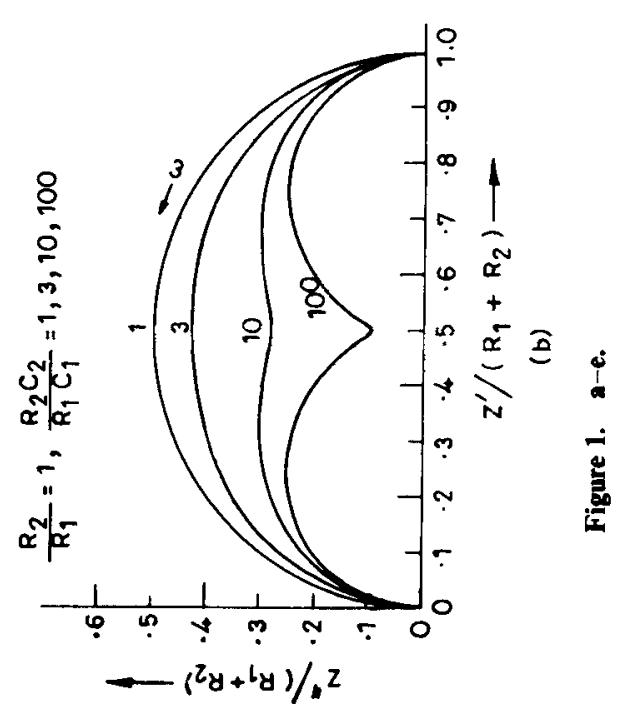



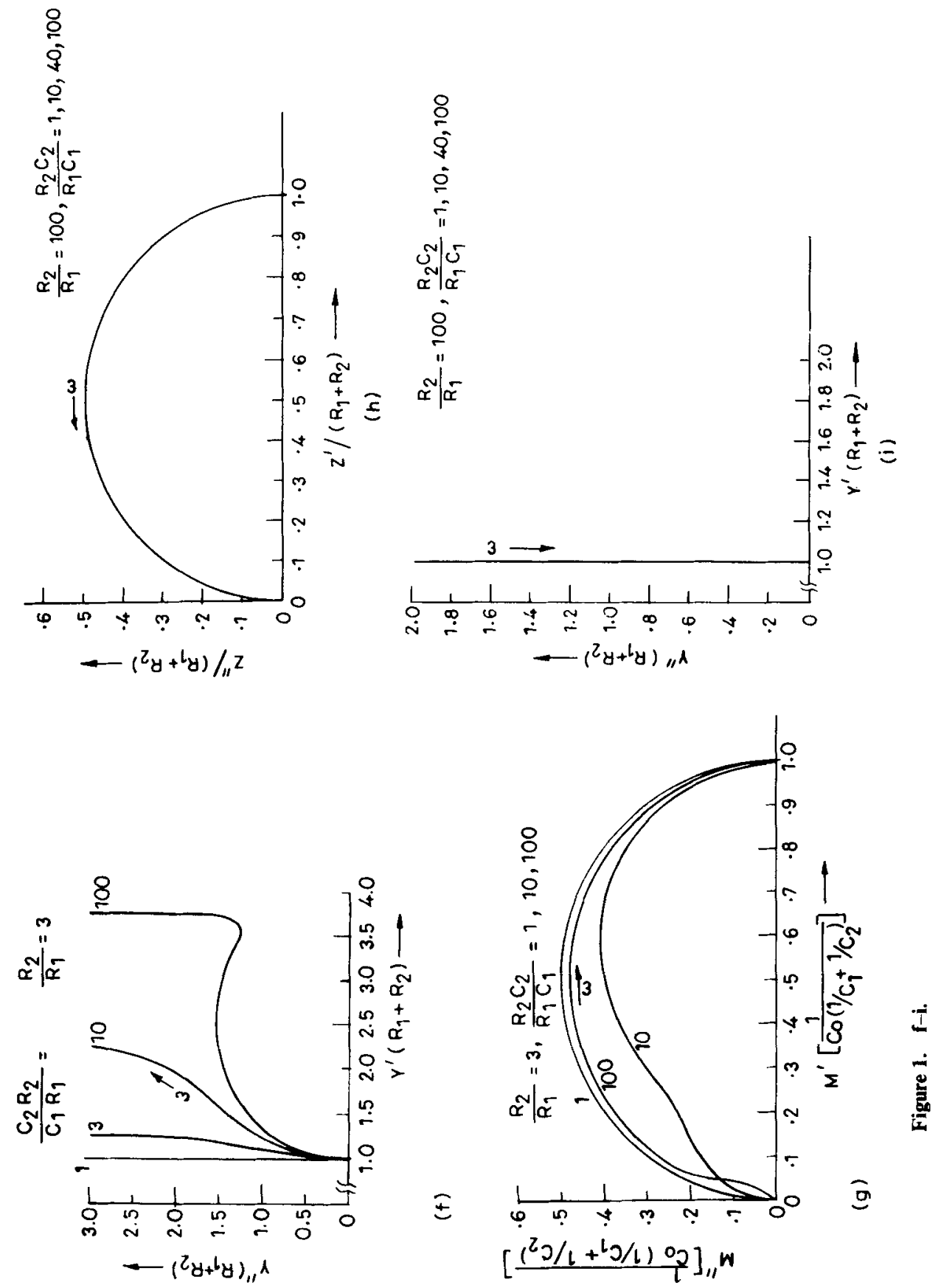


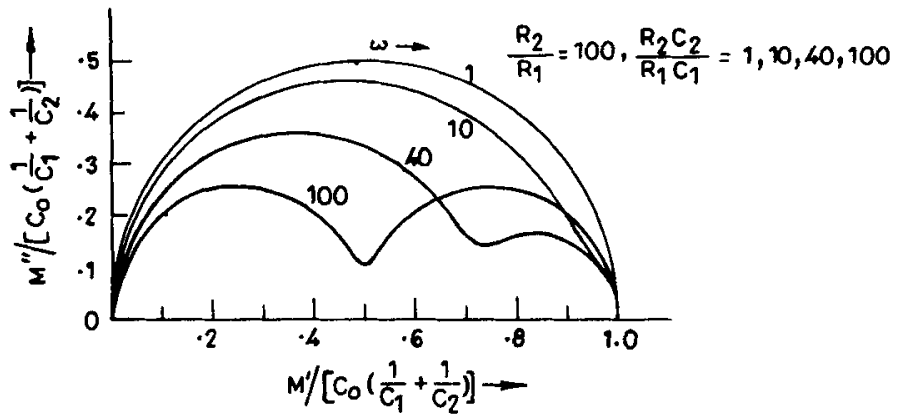

(j)
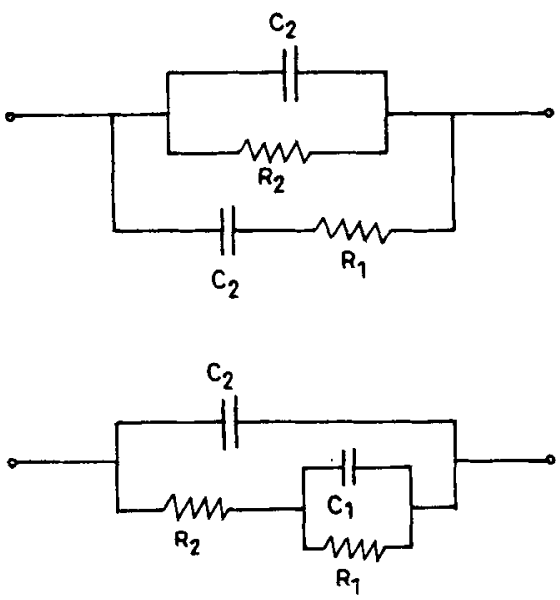

(k)

Figure 1. (a) Parallel combination of resistance, $R_{1}$ with capacitance, $C_{1}$ and resistance, $R_{2}$ with capacitance, $C_{2}$ connected in series. Normalized (b) $Z^{\prime \prime} /\left(R_{1}+R_{2}\right)$ vs $Z^{\prime} /\left(R_{1}+R_{2}\right)$, (c) $Y^{\prime \prime}\left(R_{1}+R_{2}\right)$ vs $Y^{\prime}\left(R_{1}+R_{2}\right)$ and (d) $M^{\prime \prime}\left[1 / \operatorname{Co}\left(1 / C_{1}+1 / C_{2}\right)\right]$ vs $M^{\prime}\left[1 / \operatorname{Co}\left(1 / C_{1}+1 / C_{2}\right)\right]$ plots for the circuit (a) for different $R_{2} C_{2} / R_{1} C_{2}=1,3,10$ and 100 with $R_{2} / R_{1}=1$. Normalized (e) $Z^{\prime \prime} /\left(R_{1}+R_{2}\right)$ vs $Z^{\prime} /\left(R_{1}+R_{2}\right)$ (f) $Y^{\prime \prime}\left(R_{1}+R_{2}\right)$ vs $Y^{\prime}\left(R_{1}+R_{2}\right)$ and (g) $M^{\prime \prime}\left[1 / \operatorname{Co}\left(1 / C_{1}+1 / C_{2}\right)\right]$ vs $M^{\prime}\left[1 / C o\left(1 / C_{1}+1 / C_{2}\right)\right]$ plots for the circuit (a) for different $C_{2} R_{2} / C_{1} R_{1}=1,3,10$ and 100 with $R_{2} / R_{1}=3$. (b) $Z^{\prime \prime} /\left(R_{1}+R_{2}\right)$ vs $Z^{\prime} /\left(R_{1}+R_{2}\right)$, (i) $Y^{\prime \prime}\left(R_{1}+R_{2}\right)$ vs $Y^{\prime}\left(R_{1}+R_{2}\right)$ and (j) $M^{\prime \prime} /\left[\operatorname{Co}\left(1 / C_{1}+1 / C_{2}\right)\right]$ vs $M^{\prime} /\left[\operatorname{Co}\left(1 / C_{1}+1 / C_{2}\right)\right]$ plots for $R_{2} / R_{1}=100$, and $C_{2} R_{2} / C_{1} R_{1}=1$, 10,40 and 100, (k) two similar Voigt, Maxwell and Ladder equivalent $R C$ circuits showing similar response.

The aim of this paper is to show that different combinations of RC lumped circuit elements give different shapes of complex impedance, admittance and modulus plots depending on their relative values of resistance and relaxation time. Useful information about electronic ceramic system can be obtained on comparing the experimental immittance plots with their representative circuits. The equivalent circuits and their immittance plots presented in this paper will serve as a ready reference for the above purpose by a direct comparison. The procedure is illustrated by analysing the impedance data on $\mathrm{La}_{1-x} \mathrm{Na}_{x} \mathrm{Co}_{1-x} \mathrm{Nb}_{x} \mathrm{O}_{3}(x=0.70)$ system prepared and studied in our laboratory. A preliminary report of this was presented earlier (Katare 1990). 
The general immittance functions and models are presented in $\S 2$, followed by discussion in $\S 3$. Conclusions are given in $\S 4$.

\section{Impedance functions and models}

The ac response of a material can be expressed in any of the four basic formalisms (Macdonald 1987). These are conveniently expressed as complex impedance $\left(Z^{*}\right)$, complex admittance $\left(Y^{*}\right)$, complex permittivity $\left(\varepsilon^{*}\right)$ and complex modulus $M^{*}=\left(\varepsilon^{*}\right)^{-1}$. The inter-relationship among these can be explained with the help of a lossy dielectric which can be expressed as a RC circuit. The four functions broadly called immittance functions (Macdonald 1987) are given by (2)

$$
\begin{aligned}
Z^{*} & =Z^{\prime}-j Z^{\prime \prime} ; \quad j=\sqrt{ }-1, \quad \omega=2 \pi f, \\
Y^{*} & =\left(Z^{*}\right)^{-1}=Y^{\prime}+j Y^{\prime \prime}, \\
\varepsilon^{*} & =\left(j \omega \operatorname{Co} Z^{*}\right)^{-1}=\varepsilon^{\prime}-j \varepsilon^{\prime \prime}, \\
M^{*} & =\left(\varepsilon^{*}\right)^{-1}=j \omega \operatorname{Co} Z^{*}=M^{\prime}+j M^{\prime \prime},
\end{aligned}
$$

where $f$ is the frequency of the ac excitation and $C o$ the capacitance of the empty cell used to house the material and it depends on the geometry. The study of $\varepsilon^{*}$ as a function of frequency has been widely used since the pioneering work of Cole and Cole (Von-Hipple 1954) and is most suited for dielectric materials having very low or vanishing conductivity (Macdonald 1987). For other types of materials $Z^{*}, Y^{*}$ or $M^{*}$ are more suitable as they facilitate the construction of equivalent circuits and easy visualization of various charge transfer processes. It must be noted that $Z^{*}$ and $Y^{*}$ are inversely related. Hence the frequency regions where the response of the material under test is not visible in the $Z^{*}$-plot would be highlighted in the $Y^{*}$-plot. Therefore both $Z^{\prime \prime}$ vs $Z^{\prime}$ and $Y^{\prime \prime}$ vs $Y^{\prime}$ plots should be looked at together to find an equivalent circuit representation. Similarly if there is any congestion of points in $Z^{\prime \prime}$ vs $Z^{\prime}$ or $Y^{\prime \prime}$ vs $Y^{\prime}$ plots in certain frequency ranges, $M^{\prime \prime}$ vs $M^{\prime}$ or $\varepsilon^{\prime \prime}$ vs $\varepsilon^{\prime}$ also should be looked at. A RC circuit has one time constant. It can, therefore, be conveniently used to represent one relaxation process in the dielectric material. In what follows, we give some equivalent circuit models containing four components and their $Z^{*}, Y^{*}$ and $M^{*}$ functions with their plots.

\subsection{Models involving four components}

2.1a A series combination of two parallel RC circuits is shown in figure 1a. The impedance functions $Z^{\prime}$ and $Z^{\prime \prime}$ are given by:

$$
\begin{aligned}
& Z^{\prime}=\frac{R_{1}}{1+\left(\omega C_{1} R_{1}\right)^{2}}+\frac{R_{2}}{\left(\omega C_{2} R_{2}\right)^{2}+1}, \\
& Z^{\prime \prime}=\frac{\omega C_{1} R_{1}^{2}}{\left(\omega C_{1} R_{1}\right)^{2}+1}+\frac{\omega C_{2} R_{2}^{2}}{\left(\omega C_{2} R_{2}\right)^{2}+1} .
\end{aligned}
$$

Figures $1 \mathrm{~b}-\mathrm{d}$ show normalized plots of $Z^{\prime \prime}$ vs $Z^{\prime}, Y^{\prime \prime}$ vs $Y^{\prime}$ and $M^{\prime \prime}$ vs $M^{\prime}$ for various values of the ratio of time constant. $C_{2} R_{2} / C_{1} R_{1}$ with $R_{2} / R_{1}=1$. Similar plots for $R_{2} / R_{1}=3$ are shown in figures $1 \mathrm{e}-\mathrm{g}$, for $R_{2} / R_{1}=100$ in figures $1 \mathrm{~h}-\mathrm{j}$ respectively. 

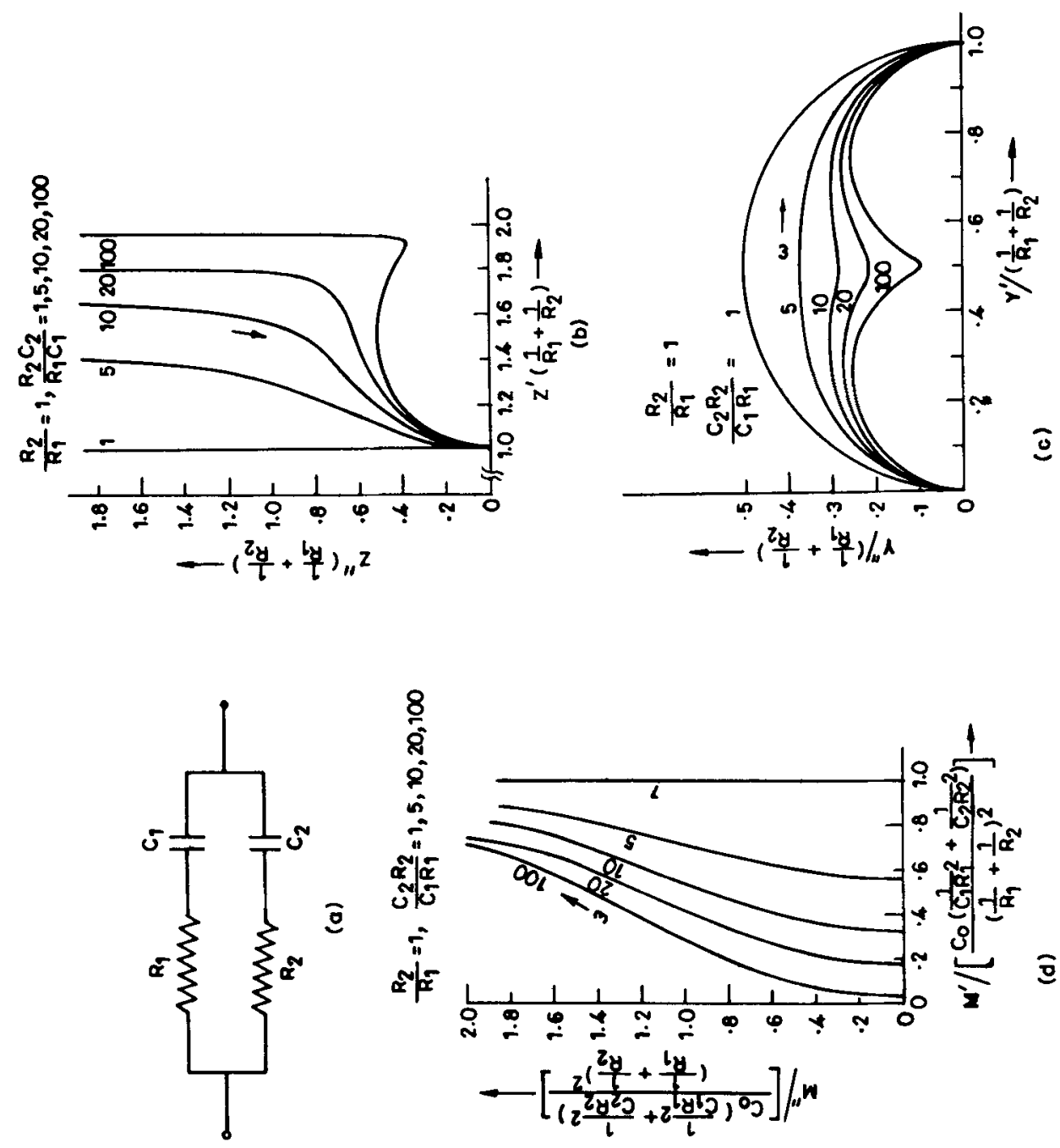

อิ 

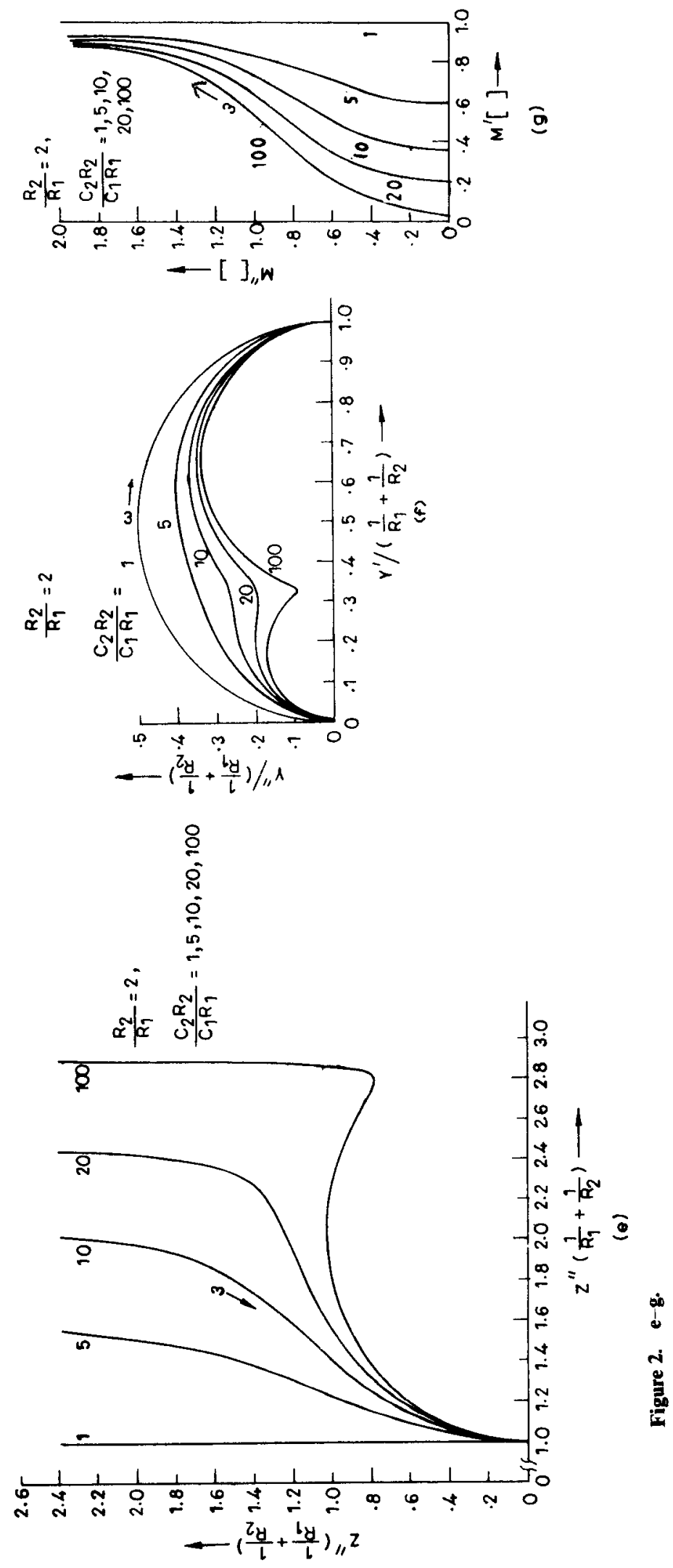

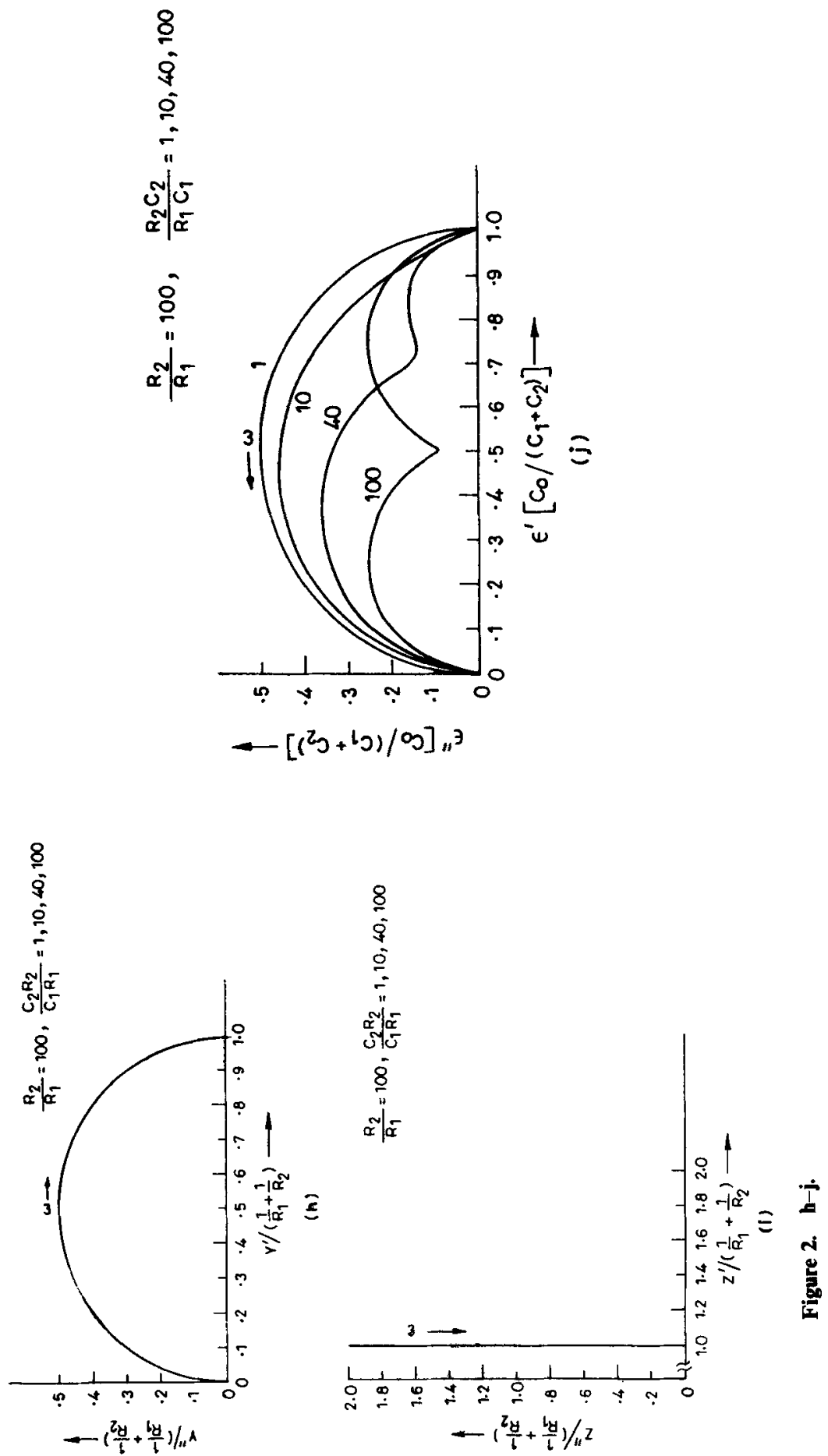

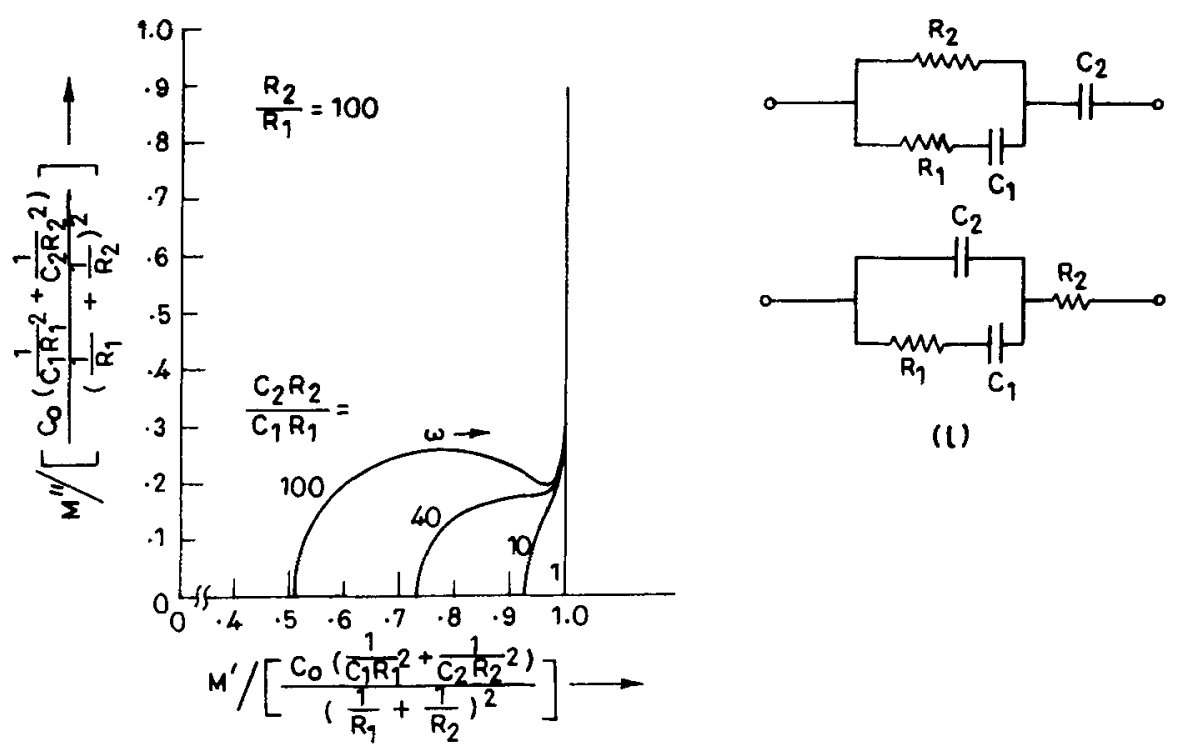

(l)

(k)

Figure 2. (a) Series combinations of resistance, $R_{1}$ with capacitance, $C_{1}$ and resistance, $R_{2}$ with capacitance, $C_{2}$ connected in parallel. Normalized (b) $Z^{\prime \prime} /\left(R_{1}+R_{2}\right)$ vs $Z^{\prime} /\left(R_{1}+R_{2}\right)$, (c) $Y^{\prime \prime}\left(R_{1}+R_{2}\right)$ vs $Y^{\prime}\left(R_{1}+R_{2}\right)$ and (d) $M^{\prime \prime}\left[1 / \operatorname{Co}\left(1 / C_{1}+1 / C_{2}\right)\right]$ vs $M^{\prime}\left[1 / \operatorname{Co}\left(1 / C_{1}+1 / C_{2}\right)\right]$ plots for the circuit (a) for different $R_{2} C_{2} / R_{1} C_{1}=1,5,10,20$ and 100 with $R_{2} / R_{1}=1$. Normalized (e) $Z^{\prime \prime} /\left(R_{1}+R_{2}\right)$ vs $Z^{\prime} /\left(R_{1}+R_{2}\right)$, (f) $Y^{\prime \prime}\left(R_{1}+R_{2}\right)$ vs $Y^{\prime}\left(R_{1}+R_{2}\right)$ and (g) $M^{\prime \prime}\left[1 / \operatorname{Co}\left(1 / C_{1}+1 / C_{2}\right)\right]$ vs $M^{\prime}\left[1 / \operatorname{Co}\left(1 / C_{1}+1 / C_{2}\right)\right]$ plots for the circuit (a) for different $C_{2} R_{2} / C_{1} R_{1}=1,5,10,20$ and 100 with $R_{2} / R_{1}=2$. (h) $Z^{\prime \prime} /\left(R_{1}+R_{2}\right)$ vs $Z^{\prime} /\left(R_{1}+R_{2}\right)$, (i) $Y^{\prime \prime}\left(R_{1}+R_{2}\right)$ vs $Y^{\prime}\left(R_{1}+R_{2}\right)$ and (j) $\varepsilon^{\prime \prime} /\left[\operatorname{Co}\left(1 / C_{1}+1 / C_{2}\right)\right]$ vs $\varepsilon^{\prime} /\left[\operatorname{Co}\left(1 / C_{1}+1 / C_{2}\right]\right.$, (k) $M^{\prime \prime} 1 / C o\left(1 / C_{1}+1 / C_{2}\right)$ vs $M^{\prime}\left[1 / C o / C_{1}+1 / C_{2}\right]$ plots for $R_{2} / R_{1}=100$ and $C_{2} R_{2} / C_{1} R_{1}=1$, 10,40 and 100 , (l) some other equivalent circuits showing similar behaviour.

Figure 1k shows two more circuits which show similar behaviour as they are equivalent. The circuits shown in figures 1a and $k$ are the so-called Voigt, Maxwell and Ladder representations (Macdonald 1987).

2.1b Another arrangement of four components $R_{1}, C_{1}$ and $R_{2}, C_{2}$ is shown in figure $2 a . Z^{\prime}$ and $Z^{\prime \prime}$ are given by:

$$
\begin{aligned}
& Z^{\prime}=\frac{\frac{\left(\omega C_{1} R_{1}\right)^{2}}{\left(\omega C_{1} R_{1}\right)^{2}+1} \cdot \frac{1}{R_{1}}+\frac{1}{R_{2}}}{\left[\frac{\left(\omega C_{1} R_{1}\right)^{2}}{\left(\omega C_{1} R_{1}\right)^{2}+1} \cdot \frac{1}{R_{1}}+\frac{1}{R_{2}}\right]^{2}+\left[\frac{\omega C_{1} R_{1}}{\left(\omega C_{1} R_{1}\right)^{2}+1} \cdot \frac{1}{R_{1}}\right]^{2}}, \\
& Z^{\prime \prime}=\frac{\frac{\omega C_{1} R_{1}}{\left(\omega C_{1} R_{1}\right)^{2}+1} \cdot \frac{1}{R_{1}}}{\left[\frac{\left(\omega C_{1} R_{1}\right)^{2}}{\left(\omega C_{1} R_{1}\right)^{2}+1} \cdot \frac{1}{R_{1}}+\frac{1}{R_{2}}\right]^{2}+\left[\frac{\left(\omega C_{1} R_{1}\right)}{\left(\omega C_{1} R_{1}\right)^{2}+1} \cdot \frac{1}{R_{1}}\right]^{2}}+\frac{1}{\omega C_{2}} .
\end{aligned}
$$

The normalized immittance plots are shown in figures $2 b-k$. Figure 21 shows another equivalent circuit which gives similar behaviour. 
2.1c A combination of four components $R_{1}, C_{1}, C_{2}$ and $C_{3}$ is shown in figure $3 \mathrm{a}$. Expressions for $Z^{\prime}, Z^{\prime \prime}$ are given below:

$$
\begin{aligned}
& Z^{\prime}=\frac{\frac{\left(\omega C_{1} R_{1}\right)^{2}}{\left(\omega C_{1} R_{1}\right)^{2}+1} \cdot \frac{1}{R_{1}}}{\left[\frac{\left(\omega C_{1} R_{1}\right)^{2}}{\left(\omega C_{1} R_{1}\right)^{2}+1} \cdot \frac{1}{R_{1}}\right]^{2}+\left[\frac{\omega C_{1} R_{1}}{\left(\omega C_{1} R_{1}\right)^{2}+1} \cdot \frac{1}{R_{1}}+\omega C_{2}\right]^{2}}, \\
& Z^{\prime \prime}=\frac{\left[\frac{\omega C_{1} R_{1}}{\left(\omega C_{1} R_{1}\right)^{2}+1} \cdot \frac{1}{R_{1}}+\omega C_{2}\right]}{\left[\frac{\left(\omega C_{1} R_{1}\right)^{2}}{\left(\omega C_{1} R_{1}\right)^{2}+1} \cdot \frac{1}{R_{1}}\right]^{2}+\left[\frac{\left(\omega C_{1} R_{1}\right)}{\left(\omega C_{1} R_{1}\right)^{2}+1} \cdot \frac{1}{R_{1}}+\omega C_{2}\right]^{2}}+\frac{1}{\omega C_{3}} .
\end{aligned}
$$

The immittance plots for their normalized values are shown in figures $3 b-d$.

\section{Discussion}

From the figures shown in $\S 2$, it is clear that different circuits will show different immittance plots. Therefore, by comparing the experimentally observed $Z^{\prime \prime}$ vs $Z^{\prime}, Y^{\prime \prime}$ vs $Y^{\prime}$ or $M^{\prime \prime}$ vs $M^{\prime}\left(\varepsilon^{\prime \prime}\right.$ vs $\left.\varepsilon^{\prime}\right)$ plots with those presented here, one can arrive at a guess about a circuit that might be used to represent electrical behaviour of the materials. However, the question that which specific equivalent circuit should be used still remains to be answered, specially in the case where the circuits shown in $\S 2$ appear to be most promising, as many equivalent circuits are possible for the same situation. The answer to this question can be obtained by employing intuition and by carrying out several impedance measurements with different conditions viz. temperature, composition, microstructure etc. (Macdonald 1987). A clue for the choice of an equivalent circuit can be obtained by comparing the experimental plots with those presented in this paper as discussed in the following paragraphs. It is always desirable to select the simplest of the possible equivalent circuits (Sinclair and West 1989).

As an example, we consider the circuit containing two parallel $R C$ elements connected in series as shown in figure 1a. $Z^{\prime \prime}$ vs $Z^{\prime}$ plots for various values of $R_{2} / R_{1}$ and $R_{2} C_{2} / R_{1} C_{1}$ indicate that when values of $R_{2} C_{2}$ and $R_{1} C_{1}$ are quite different from each other, well resolved arcs appear. If the experimental observations made for an electronic ceramic show such a behaviour, then we can represent the material by such an equivalent circuit and the relative values of $R_{1}, R_{2}$ and $R_{1} C_{1}, R_{2} C_{2}$ can be estimated by comparing the experimental curves with those presented in figure $1 \mathrm{~b}$. This can in turn be used to infer that two distinct charge transfer processes are present in the material. An interesting situation arises when the time constants $R_{1} C_{1}$ and $R_{2} C_{2}$ are quite different from each other and $R_{2}, R_{1}$ are also quite different. Plot of $Z^{\prime \prime}$ vs $Z^{\prime}$ shown in figure $1 \mathrm{~h}$ for this situation $\left(R_{2} / R_{1}=100, C_{2} R_{2} / C_{1} R_{1}=100\right)$ has single circular arc which is the same for a circuit having a single parallel combination of $R$ and $C$.

Actually in this case also two distinct arcs appear but since $R_{2}$ and $R_{1}$ are quite different, the $Z^{\prime \prime}$ vs $Z^{\prime}$ plot contains a big arc and a tiny arc which are masked by the bigger ones. The $Z^{\prime \prime}$ vs $Z^{\prime}$ plot is thus dominated by the bigger $R$. Similar is the situation with $Y^{\prime \prime}$ vs $Y^{\prime}$ plot. It shows a vertical straight line exactly similar to the case of a single RC parallel combination. 
Therefore, if experimental observations of $Z^{\prime \prime}$ and $Z^{\prime}$ for an electronic ceramic show a single circular arc, then one might be tempted to say that the appropriate model for this material is a parallel combination of $R$ and $C$ (see figures $1 \mathrm{~h}$ and $\mathrm{i}$ ) and a single process is present. However, this may be wrong conclusion. This problem can be solved by using other immittance plots. Figure $1 \mathrm{j}$ shows $M^{\prime \prime}$ and $M^{\prime}$ plot for such a situation with $R_{2} / R_{1}=100$ and $R_{2} C_{2} / R_{1} C_{1}=100$. Clearly two arcs appear and presence of two parallel $\mathrm{RC}$ circuits connected in series and hence presence of two processes is indicated. So, for the cases where single arc appears in the experimental $Z^{\prime \prime}$ vs $Z^{\prime}$ plots, other immittance plots should also be looked at. It should be noted that a particular value of $R_{2} C_{2} / R_{1} C_{1}$ can be obtained by several combinations of $R_{2} / R_{1}$ and $C_{2} / C_{1}$. If $R_{2} C_{2} / R_{1} C_{1}$ is a large number (say 100 ), two interesting cases are (i) $R_{2} / R_{1}=100$, $C_{2} / C_{1}=1$ and (ii) $R_{2} / R_{1}=1, C_{2} / C_{1}=100$. In case (i), $M$ plots would be useful whereas in case (ii) $Z$ plots would be useful (Sinclair and West 1989). Also as indicated in figure 2 , sometimes $\varepsilon$ plots would be more revealing. For this case, $Z, Y$ and $M$ plots show the presence of single process whereas $\varepsilon$ plot shows the presence of two processes.

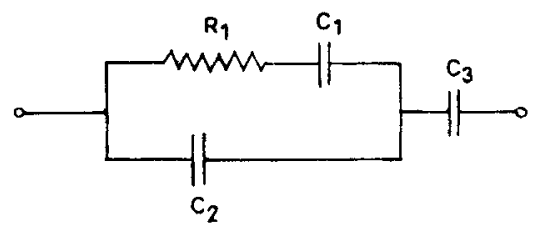

(a)

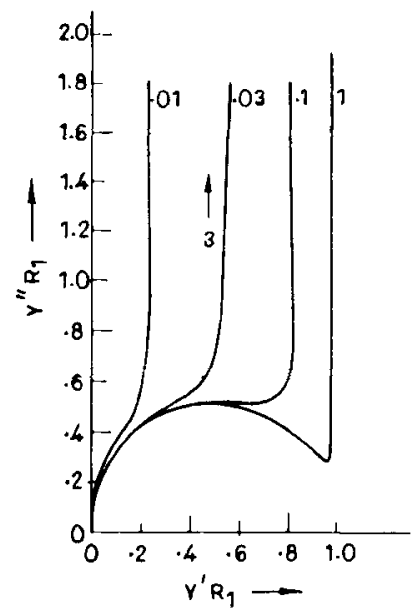

(c)

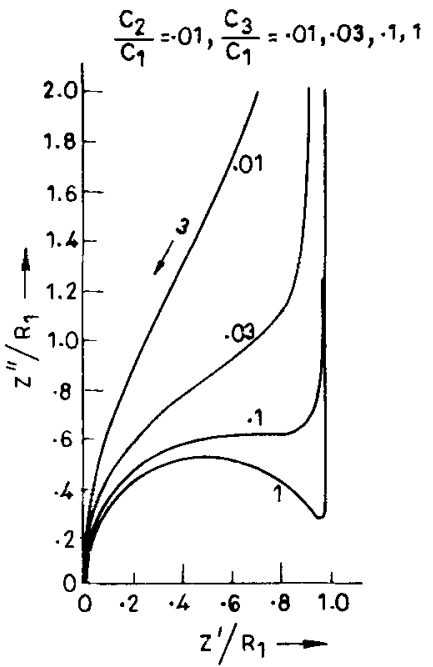

(b)

Figure 3. a-c. 


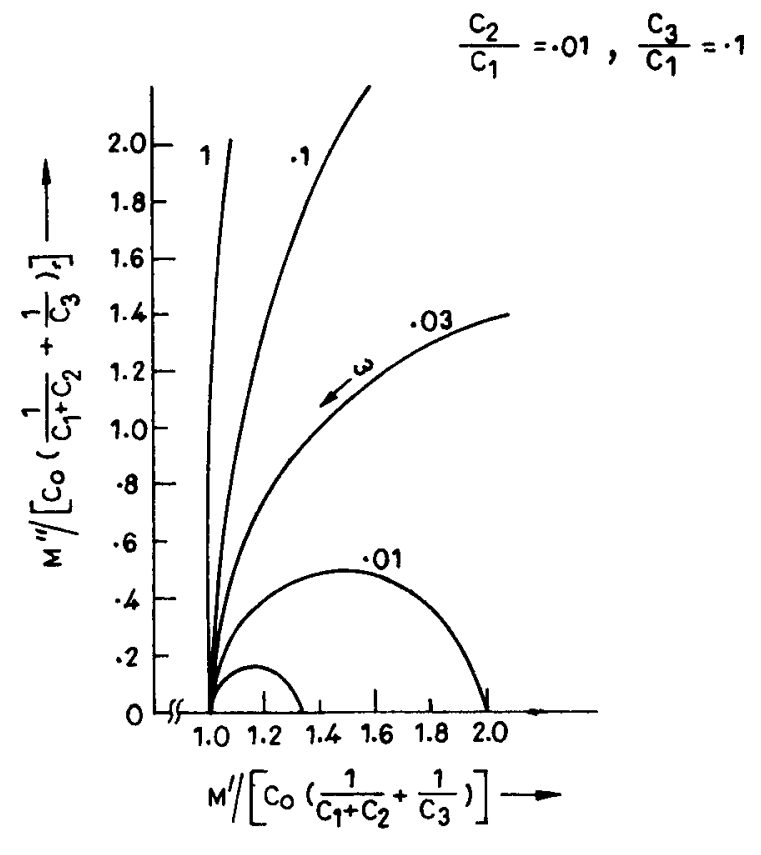

(d)

Figure 3. (a) A circuit where series combination of $R_{1}$ and $C_{1}$ connected in parallel to $C_{2}$ is connected in series with $C_{3}$, (b) $Z^{\prime \prime} / R_{1}$ vs $Z^{\prime} / R_{1}$, (c) $Y^{\prime \prime} R_{1}$ vs $Y^{\prime} R_{1}$ and (d) $M^{\prime \prime}\left[1 / C o\left(1 / C_{1}+1 / C_{2}\right)\right]$ vs $M^{\prime}\left[1 / C o\left(1 / C_{1}+1 / C_{2}\right)\right]$ plots for $C_{2} / C_{1}=0 \cdot 01 . C_{3} / C_{1}=0 \cdot 01$, $0-03,0 \cdot 1$ and 1 .

This analysis when extended to include more than two parallel $R C$ combinations connected in series reveals that whenever the time constants are well separated distinct arcs appear, the number of arcs being equal to the number of $R C$ combinations and when they are close to each other (within a factor of 5), a single depressed looking arc results. This situation for the case of two-parallel $R C$ combination connected in series is shown in figures $1 \mathrm{~b}-\mathrm{f}$. Therefore, whenever depressed circular arcs appear in immittance plots for a material, presence of at least two time constants (i.e. processes) close to each other is indicated. A usual practice in such cases is to represent the material by a circuit having a single parallel combination of $R$ and $C$ and say that there exists a distribution of time constants centred around the value of $R C$. On this issue, use of equivalent circuits containing constant phase angle elements capable of producing depressed arcs have been recommended (Armstrong and Burnham 1976; Macdonald 1987) to represent the behaviour of the material. The physical origin of the behaviour such that it can be modelled by constant phase angle elements has also been sought out (Jonscher 1977, 1983, 1990).

We now present a preliminary analysis of the results obtained for the system $\mathrm{La}_{1-x} \mathrm{Na}_{x} \mathrm{Co}_{1-x} \mathrm{Nb}_{x} \mathrm{O}_{3}(x=0 \cdot 70)$ in our laboratory. Figure 4 shows $Z^{\prime \prime}$ vs $Z^{\prime}$ and $Y^{\prime \prime}$ vs $Y^{\prime}$ plots for this system at $430 \mathrm{~K}$. Similar plots were obtained at other temperatures. A comparison with figures corresponding to circuits in $\$ 2.1$ reveals that the model circuit needed to represent this system would be similar to the one shown in figure 1a i.e. two parallel $R C$ combinations connected in series. The representative values of $R_{1}$, $C_{1}, R_{2}$ and $C_{2}$ obtained from intercepts are approximately equal to $4 \cdot 75 \times 10^{3} \Omega$, 


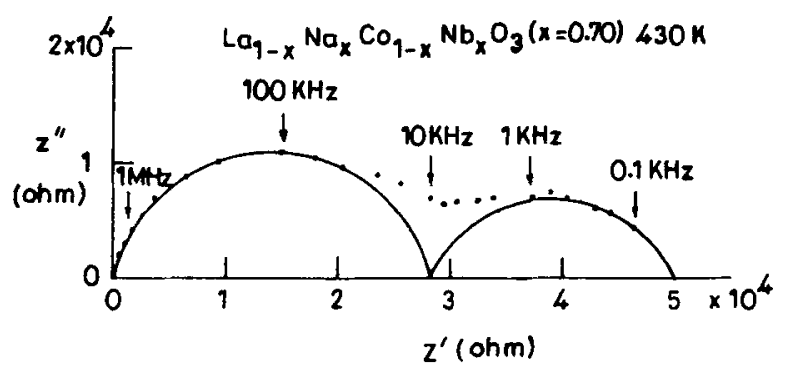

(a)
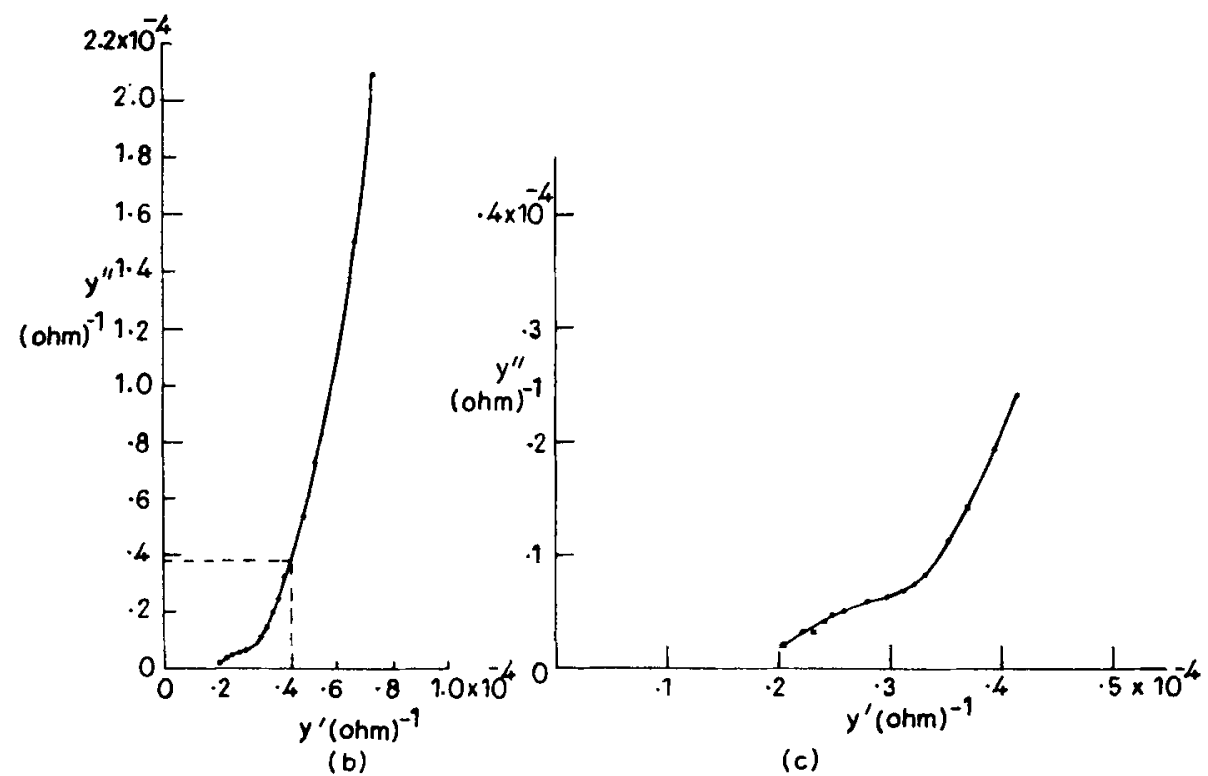

(b)

(c)

Figure 4. (a)-(c) $Z^{\prime \prime}$ vs $Z^{\prime}$ and $Y^{\prime \prime}$ vs $Y^{\prime}$ plots for the $\mathrm{La}_{1-x} \mathrm{Na}_{x} \mathrm{Co}{ }_{1-x} \mathrm{Nb}_{x} \mathrm{O}_{3}(x=0.70)$ ceramic system at $430 \mathrm{~K}$.

$4.8 \times 10^{-11} \mathrm{~F}, 3.2 \times 10^{3} \Omega$ and $1.6 \times 10^{-8} \mathrm{~F}$ at $493 \mathrm{~K}, 2.75 \times 10^{4} \Omega, 5.8 \times 10^{-11} \mathrm{~F}$, $2.2 \times 10^{4} \Omega$ and $1.0 \times 10^{-8} \mathrm{~F}$ at $430 \mathrm{~K}$ and equal to $4.75 \times 10^{6} \Omega, 4.8 \times 10^{-12} \mathrm{~F}$, $4.25 \times 10^{6} \Omega$ and $3.7 \times 10^{-10} \mathrm{~F}$ at $357 \mathrm{~K}$ respectively. Since the arcs are depressed there is a distribution of time constants or at least two time constants for each depressed arc. In ceramics, usually we have grain, grain-boundary and contact electrode contributions to the overall measured properties. So, in general, we expect the presence of three arcs. It is very likely that grain and grain-boundary processes are having time constants close to each other giving rise to the left arc which is very little depressed and the arc appearing at lower frequencies corresponds to the electrodes. By performing the experiment with different electrodes, the contact electrode contributions can be separated out. These results will be presented elsewhere.

It is to be mentioned that the values of the $R$ and $C$ elements obtained by comparing the experimental data with the simulated diagrams are highly approximate. A great deal of improvement in the accuracy of the values can be achieved by using a simultaneous fitting of the immittance values using a multivariable non-linear least squares 
procedure (Macdonald and Garber 1977; Macdonald et al 1982; Macdonald 1987). A preliminary report of the numerical scheme to achieve this-developed in our laboratory-was given earlier (Pandey 1992, 1993).

In general, then, the procedure that we propose to be adopted for the complex impedance analysis of electronic ceramics is that, first, the experimental plots should be compared with the simulated diagrams reported in 2 , thereby obtaining a guess for the most appropriate type of model. Next, if the arcs are depressed then a distribution of time constants or presence of at least two or three constants may be assumed and all the parameters determined accurately using a least squares procedure. Great attention should be paid to the behaviour of the sample holder being used as the sample holder itself might create an artifact (Sayer et al 1992; Pandey 1993). A detailed analysis of this will be presented elsewhere (Pandey et al 1995).

\section{Conclusion}

In this paper we have presented the simulated diagrams of $Z^{\prime \prime}$ vs $Z^{\prime}, Y^{\prime \prime}$ vs $Y^{\prime}$ and $M^{\prime \prime}$ vs $M^{\prime}$ for model circuits containing four elements. An example for analysing the complex immittance data for the given electronic system $\mathrm{La}_{1-x} \mathrm{Na}_{x} \mathrm{Co}_{1-x} \mathrm{Nb}_{x} \mathrm{O}_{3}$ is presented. A direct comparison of the experimentally obtained impedance curves with those of model equivalent circuit yields a correct guess for the type of equivalent circuit to be pursued. With these initial guesses, further improvement in their values and their distributions can be obtained using least squares fitting procedure. Finally the various circuit elements have to be identified with different electrical charge transfer processes in the materials.

\section{References}

Armstrong R D and Burnham R A 1976 J. Electroanal. Chem. 72257

Gerhardt R and Nowick A S 1986 J. Am. Ceram. Soc. 69641

Hodge I M, Ingram M D and West A R 1976 J. Electroanal. Chem. 74125

Hooper A 1977 J. Phys. D: Appl. Phys. 101487

Jonscher A K 1977 Nature 267673

Jonscher A K 1983 in Dielectric relaxation in solids (London: Chelsea Dielectric Press)

Jonscher A K 1990 Phys. Thin Films 11205

Katare R K 1990 in Modelling of dielectric properties of materials, M. Phil dissertation, Rani Durgavati University, Jabalpur

Macdonald J R (ed.) 1987 in Impedance spectroscopy (New York: John Wiley and Sons)

Macdonald J R and Garber J A 1977 J. Electrochem. Soc. 1241022

Macdonald J R, Schoonman J and Lehnen A P 1982 J. Electroanal. Chem. 13177

Pandey L 1992 in Workshop on use of computers in teaching physics, Jabalpur

Pandey L 1993 in Discussion meeting on ferroelectric ceramics, Varanasi

Pandey L, Parkash O, Tewari H S, Thakur O P and Kumat D 1993 Materials Res. Soc. India (MRSI) Meeting. Trivandrum

Pandey L, Parkash O and Kumar D 1995 Indian J. Pure Appl. Phys. (in press)

Parkash O, Pandey L, Tewari H S, Tare V B and Kumar D 1990 Ferroelectrics 102203

Sayer M, Mansingh A, Arora A K and Lo A 1992 Integrated Ferroelectrics 1129

Sinclair D C and West A R 1989 J. Appl. Phys. 663850

Von Hipple R 1954 in Dielectric and waves (New York: John Wiley and Sons) 